\title{
LDL acts as an opsonin enhancing the phagocytosis of group $A$ Streptococcus by monocyte and whole human blood
}

\author{
Lulei Zhou ${ }^{1,2} \cdot \operatorname{Ling} \mathrm{Liu}^{1,2} \cdot$ Jinli Yang ${ }^{1,2} \cdot$ Yuxin Li $^{1,2} \cdot$ Wencheng Bai ${ }^{1,2} \cdot \mathrm{Na} \mathrm{Liu}^{1,2} \cdot$ \\ Wenlong $\mathrm{Li}^{1,2} \cdot$ Yumin Gao ${ }^{1,3} \cdot$ Liping $\mathrm{Xu}^{1,4} \cdot \mathrm{Zhi} \mathrm{Liu}^{1,2} \cdot$ Runlin Han $^{1,2}$
}

Received: 28 May 2015 / Accepted: 6 September 2015 / Published online: 21 September 2015

(C) The Author(s) 2015. This article is published with open access at Springerlink.com

\begin{abstract}
Low-density lipoprotein (LDL) binds to group A Streptococcus (GAS) through Sc11 protein, and scavenger receptor CD36 of monocyte mediates the endocytosis of native or modified LDL. Therefore, we hypothesized that LDL might be an opsonin enhancing the phagocytosis of LDL-bound GAS by monocyte. The results showed that LDL could significantly promote U937 cell to phagocytose M28 (ATCC BAA1064) and M41 (ATCC 12373, AM41)type GAS, and the phagocytosis rates were significantly increased, compared with LDL-free group. LDL, however, did not enhance the phagocytosis of M41 (CMCC 32198, CM41) or M6 (ATCC BAA946)-type GAS since these two strains did not bind to LDL. CD36 was the major scavenger receptor mediating the uptake of LDL-bound GAS by monocyte U937 cells since anti-CD36 antibody abolished
\end{abstract}

Lulei Zhou and Ling Liu have contributed equally to this work.

Electronic supplementary material The online version of this article (doi:10.1007/s00430-015-0436-8) contains supplementary material, which is available to authorized users.

Runlin Han

han-runlin@163.com

1 Research Center of Plasma Lipoprotein Immunology, Inner Mongolia Agricultural University, Huhhot, China

2 Key Laboratory of Animal Clinic Diagnosis and Treatment, Ministry of Agriculture of China, Inner Mongolia Agricultural University, Huhhot, China

3 Present Address: College of Public Health, Inner Mongolia Medical University, Huhhot, China

4 Present Address: College of Basic Medicine, Inner Mongolia Medical University, Huhhot, China the phagocytosis of LDL-opsonized GAS but anti-CD4 antibody did not. Most of AM41-type GAS cells were killed in human blood, whereas only a few CM41-type cells were phagocytosed. Moreover, recombinant Scl1 (rScl1) derived from M41-type GAS could significantly decrease the opsonophagocytosis of AM41 but not CM41-type GAS because the rScll competitively blocked the binding of AM41-type GAS to LDL. Therefore, our findings suggest that LDL may be an opsonin to enhance CD36-dependent opsonic phagocytosis of GAS by monocyte.

Keywords Low-density lipoprotein - Group A Streptococcus · Opsonin · Phagocytosis · Monocyte

\author{
Abbreviations \\ GAS Group A Streptococcus \\ AM41 M41-type GAS (ATCC 12373) \\ CM41 M41-type GAS (CMCC 32198) \\ LDL Low-density lipoprotein
}

\section{Introduction}

Streptococcus pyogenes [group A Streptococcus (GAS)] can cause a number of suppurative infections, including pharyngitis, impetigo/pyoderma, erysipelas, cellulitis, necrotizing fasciitis, toxic streptococcal syndrome, and scarlet fever, as well as nonsuppurative sequelae including acute rheumatic fever and acute glomerulonephritis [1]. Based on the surface M protein, GAS is serologically separated into over $200 \mathrm{M}$ protein serotypes [2]. Streptococcal collagen-like protein 1 (Scl1) as one of the virulence factors of GAS is found on the cell surface of many M-type GAS [3]. Although Scl1s expressed by different M-type GAS share similar triple helix structure their amino acid 
sequence, especially in variable $\mathrm{N}$-terminal region, varies significantly, so that Scl1s in M6 and M55-type GAS specifically bind to factor H [4], whereas Scl1s in M1, M12, M28 and M41 can interact with low-density lipoprotein (LDL) [5]. However, the significance of Scl1-LDL interaction remains unknown.

HDL and LDL especially OxLDL were found to play anti-infectious role in protecting bacteria, viruses, or parasites infections in some studies, regardless of some contradicting findings in other studies [6]. Scavenger receptor CD36 mediates the uptake of modified and native LDL and HDL by monocyte [7, 8]; however, OxLDL upregulates CD36, whereas HDL downregulates CD36 via PPARmediated pathways [7].

Therefore, we hypothesize that LDL may be an opsonin to interact with Scl1 to enhance the phagocytosis of LDLbound GAS by monocyte/macrophage.

\section{Materials and methods}

\section{Bacterial cultures and fluorescence labeling}

Three GAS strains M6 (ATCC BAA946), M28 (ATCC BAA1064), and M41 (ATCC 12373, AM41) were purchased from American Tissue Culture Collection, and one strain M41 (CMCC 32198, CM41) was obtained from China Medical Culture Collection Center, respectively. Scll nucleotide sequence of CM41 is the same as that of AM41 (GenBank: EU915249.1). Some characteristics of four GAS strains are listed in Table 1. Scl1 expression and LDL binding capacity of the four GAS strains were assayed using RT-PCR and ELISA as described previously [5]. Based upon RT-PCR analysis, scl1 gene was expressed in AM41-type GAS but not in natural mutant CM41-type GAS (Figure S1A). Moreover, AM41-type GAS could bind to LDL but the interaction of CM41-type GAS with LDL was weak as demonstrated with ELISA (Figure S1B).

GAS cultures were grown on brain-heart infusion agar (BD Biosciences, USA) overnight and in Todd Hewitt broth (BD Biosciences, USA) supplemented with $0.2 \%$ yeast extract (THY medium) at $37{ }^{\circ} \mathrm{C}$ in an atmosphere of $5 \% \mathrm{CO}_{2}$. Logarithmic phase cultures harvested at the optical density $(600 \mathrm{~nm})$ of about $0.5-0.6($ OD600 $\mathrm{nm})$ were

Table 1 Characteristics of GAS strains

\begin{tabular}{lll}
\hline GAS strain & Scl1 expression & Binding to LDL \\
\hline M41 (ATCC 12373, AM41) & + & + \\
M41 (CMCC 32198, CM41) & - & - \\
M28 (ATCC BAA1064) & + & $+[5]$ \\
M6 (ATCC BAA946) & + & $-[5]$ \\
\hline
\end{tabular}

used to prepare GAS. Colony counts were verified by plating on THY agar.

Prior to use in uptake assays, bacteria were labeled with fluorescein isothiocyanate (FITC, Sigma, USA) using method described previously [9]. Briefly, $10 \mathrm{ml}$ of exponentially growing bacterial culture was pelleted and washed twice with sterile phosphate-buffered saline (PBS). The bacterial pellet was resuspended in $1 \mathrm{ml}$ labeling solution $(0.2 \mathrm{mg} / \mathrm{ml}$ FITC in PBS) and incubated in an atmosphere of $5 \% \mathrm{CO}_{2}$ at $37{ }^{\circ} \mathrm{C}$ for $30 \mathrm{~min}$. The FITC-labeled bacteria were pelleted, washed once with cold PBS, and resuspended in RPMI 1640 medium (Gibco, USA) at a final concentration of $\sim 10^{9} \mathrm{cfu} / \mathrm{ml}$.

\section{Recombinant protein}

Recombinant Scl1 (rScl1, C176) derived from M41-type GAS was produced in E. coli using the Strep-tag II expression and purification system (IBA-GmbH, Germany) as described previously $[5,10]$. Briefly, six milliliter overnight pre-cultures of $E$. coli BL21 was inoculated into $300 \mathrm{ml}$ of Luria-Bertani (LB) broth containing $100 \mu \mathrm{g} / \mathrm{ml}$ ampicillin and incubated at $37{ }^{\circ} \mathrm{C}$ under agitation $(200 \mathrm{rpm})$. When OD600 reached $0.5-0.6$, protein expression was induced by addition of anhydrotetracycline $(0.2 \mu \mathrm{g} / \mathrm{ml})$ and incubated at $30{ }^{\circ} \mathrm{C}$ under agitation $(200 \mathrm{rpm})$ for another $3 \mathrm{~h}$. Bacteria were harvested by centrifugation $\left(10,000 \times g, 10 \mathrm{~min}, 4^{\circ} \mathrm{C}\right)$, and the pellets were resuspended in a lysis buffer $(100 \mathrm{mM}$ Tris-HCl, $1 \mathrm{mM}$ EDTA pH 8.0, $500 \mathrm{mM}$ sucrose, $0.2 \mathrm{M}$ PMSF) containing $10 \mu 1$ lysozyme and $20 \mu 1$ DNase. After incubated at room temperature for $30 \mathrm{~min}$, the lysates were clarified by centrifugation $\left(10,000 \times g, 10 \mathrm{~min}, 4^{\circ} \mathrm{C}\right)$, and the recombinant polypeptides in supernatant were purified by affinity chromatography with Strep-Tactin Sepharose.

The protein content in the elution was determined by BCA $^{\mathrm{TM}}$ Protein Assay Kit (Pierce Biotechnology, USA) using bovine serum albumin as a standard. Endotoxin in rScl1 preparations was removed by high-capacity endotoxin removal resin (Pierce Biotechnology, USA). The endotoxin content of rScl1 solution was $<3 \mathrm{EU} / \mathrm{ml}$ determined by LAL Chromogenic Endotoxin Quantitation Kit (Pierce Biotechnology, USA).

\section{Cell cultures}

Most of the macrophage scavenger receptors, including class A, B, D, E, F and G, can mediate the internalization of LDL and/or oxLDL, whereas only class B receptors, including Type I, Type II and CD36, present on monocyte [7]. Therefore, human monocytic tumor cell line U937 was used as a simple model to explore LDL-mediated opsonophagocytosis in the current study. Human monocytic U937 cells (ATCC CRL-1593.2, USA) were maintained 
in RPMI1640 medium supplemented with $10 \%$ heatinactivated fetal calf serum (Gibco, Australia), $292.28 \mu \mathrm{g} /$ $\mathrm{ml} \mathrm{L-glutamine} \mathrm{and} 100$ units $/ \mathrm{ml}$ penicillin/streptomycin (Gibco, USA). In the phagocytosis assays, cells were harvested and re-suspended in RPMI 1640 medium at a final concentration of $2.5 \times 10^{6} / \mathrm{ml}$.

\section{In vitro phagocytic assay}

\section{LDL-mediated phagocytic assay}

For opsonophagocytosis experiments, $100 \mu \mathrm{l}$ of FITClabeled GAS $\left(\sim 10^{9} \mathrm{cfu} / \mathrm{ml}\right)$ with or without LDL $(10 \mu \mathrm{g} /$ $\mathrm{ml}$ final concentration of protein) (Biomedical Technologies, USA) was added into $1 \mathrm{ml}$ of U937 cells $\left(2.5 \times 10^{6} \%\right.$ $\mathrm{ml}$ ) and incubated at $37{ }^{\circ} \mathrm{C}$ in an atmosphere of $5 \% \mathrm{CO}_{2}$. The final bacteria/monocytes ratio was about 15-50:1. The viable bacterium number in the mixture was determined by plating diluted samples onto THY agar plates after 15-, 30-, 60-min incubation. To monitor bacterial fluorescence intensity after the incubation, $200 \mu \mathrm{l}$ of sample was pipetted into the 96-well microplate and measured at $\lambda=495 \mathrm{~nm}$ (excitation) and $\lambda=519 \mathrm{~nm}$ (emission) using a fluorescence plate reader (model Synergy HT, BioTek, USA).

To test the influence of LDL concentration on opsonophagocytosis, 10,100 or $1000 \mu \mathrm{g} / \mathrm{ml}$ of LDL was used. To study the competitively inhibitory effect of rScl1 on the LDL-mediated phagocytosis, assays were performed in essentially the same manner as described above except LDL was pre-incubated with an equal mole $(20 \mathrm{nmol})$ of rScl1 for $1 \mathrm{~min}$ before adding to the cell culture.

To determine whether scavenger receptor CD36 was involved in the LDL-mediated phagocytosis of GAS by monocyte U937, monoclonal anti-CD36 antibody (SMO, Ancell, USA) was added to the cell suspension $(10 \mu \mathrm{g} / \mathrm{ml}$ final concentration) to block the receptor before the phagocytosis assay. Irrelevant monoclonal anti-CD4 antibody (Ancell, USA) was used as the negative control $(10 \mu \mathrm{g} / \mathrm{ml}$ final concentration).

\section{Microscopy}

In above LDL-mediated phagocytic assay, a $100 \mu \mathrm{l}$ aliquot of U937 cells and FITC-labeled GAS mixture was taken out after 30-min incubation and placed on ice. DAPI (Sigma, USA) was added at a final concentration of $10 \mu \mathrm{g} /$ $\mathrm{ml}$. After 10-min incubation, five milliliters of sample was plated onto slides, and FITC-GAS and DAPI-stained U937 cell nuclei were imaged at $200 \times$ magnification on fluorescent microscope (model BX41, Olympus, Japan) equipped with MicroPublisher 3.3 RTV camera (Qimaging, Canada). After acquisition, two photographs of GAS (green) and nuclei (blue) in the same field of view were merged using Image Pro Plus 6.0 software.

\section{Ex vivo phagocytic assay with human blood}

Blood was obtained from healthy volunteers in accordance with Inner Mongolia Agricultural University institutional regulations. Whole blood was collected into $5-\mathrm{ml}$ pyrogen-free vacuum tubes containing $3.2 \%$ sodium citrate as anticoagulant. A $200 \mu \mathrm{l}$ aliquot of blood was pre-incubated with $50 \mu \mathrm{rScl} 1$ or PBS at $37{ }^{\circ} \mathrm{C}$ under agitation $(170 \mathrm{rpm})$ for $1 \mathrm{~min}$. A $100 \mu \mathrm{l}$ aliquot of AM41-type or CM41-type GAS $\left(\sim 10^{3} \mathrm{cfu} / \mathrm{ml}\right)$ was added into the blood and agitated for $15 \mathrm{~min}$ at $37{ }^{\circ} \mathrm{C}$. The cfu was determined by plating samples on THY agar plates.

\section{Ethics statement}

Experiments with human blood were approved by Academic Board and Science and Technology Department of the Inner Mongolia Agricultural University. The blood was taken from the volunteers with their authorization. All adult subjects signed written informed consent form, and children were not invited to participate in this project.

\section{Statistics}

All treatments were performed in triplicate, and all experiments were conducted for three times. All data are presented as the mean \pm SD. Statistical significance was performed using the two-tailed paired Student's $t$ test. Significance was determined at a level of $[p<0.001(* * *)]$.

\section{Results}

\section{LDL promotes the phagocytosis of GAS by human U937 monocyte}

To test whether LDL can promote the phagocytosis of GAS by monocyte, colony counting, fluorescence intensity detection and microscopy observation were employed (Fig. 1). Based upon colony counts, LDL significantly enhanced the phagocytosis of M41-type GAS (ATCC 12373, AM41-type GAS). The phagocytosis rates were increased by 17,58 and $48 \%$ at 15,30 and $60 \mathrm{~min}$, respectively, compared with LDL-free control ( $p<0.01$, Fig. 1a3). Similar results were obtained with fluorescence intensity detection. The uptake efficiency was increased by 17.6, 26.6 and $23.6 \%$ at 15,30 and $60 \mathrm{~min}$, respectively, compared with LDL-free control (Fig. 1a3). The phagocytosis rates determined by fluorescence intensity were usually lower than those calculated 


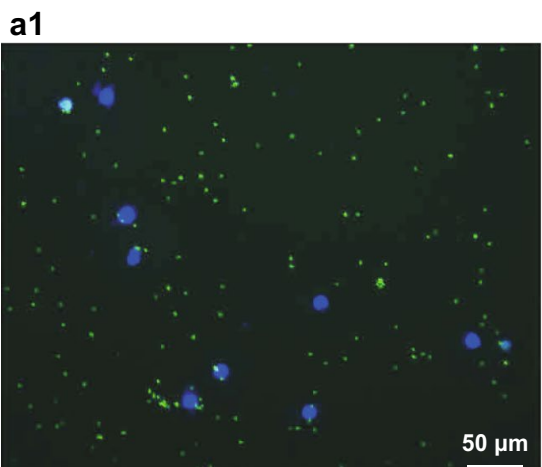

AM41+U937, $30 \mathrm{~min}$

a1

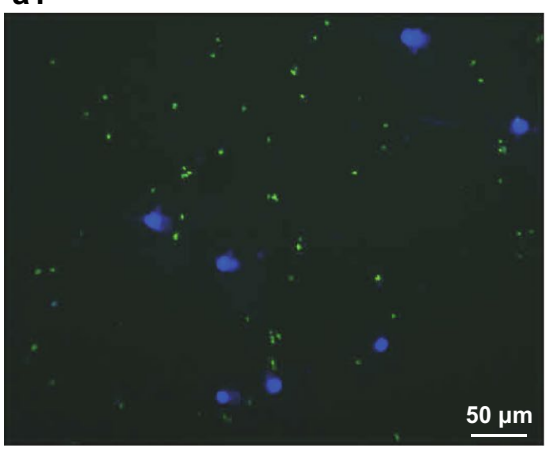

CM41+U937, 30 min

c1

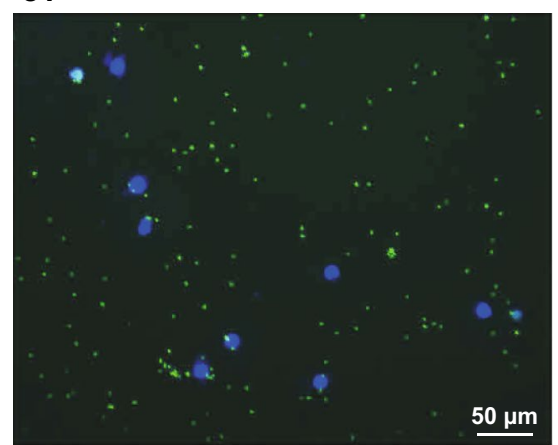

M28+U937, $30 \mathrm{~min}$

d1

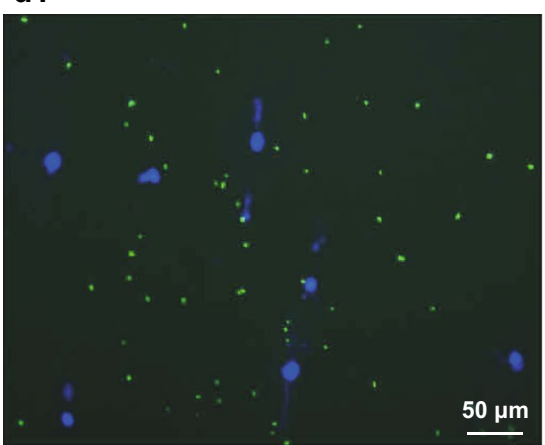

M6+U937, $30 \mathrm{~min}$ a2

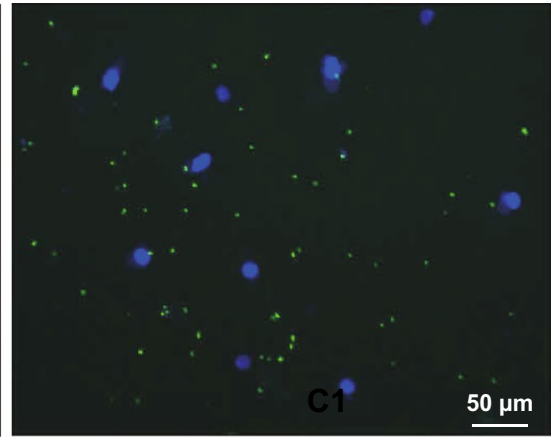

AM41+U937+LDL, 30 min

a2

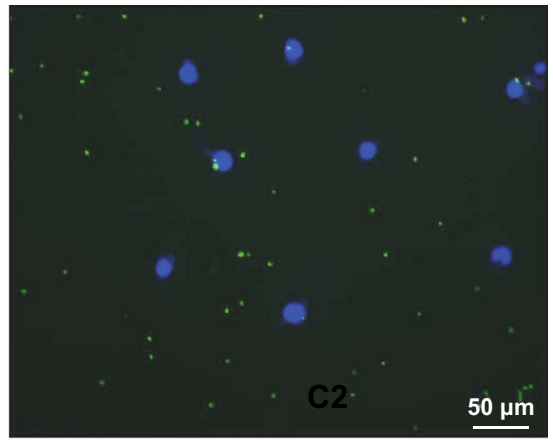

CM41+U937+LDL, 30 min

c2

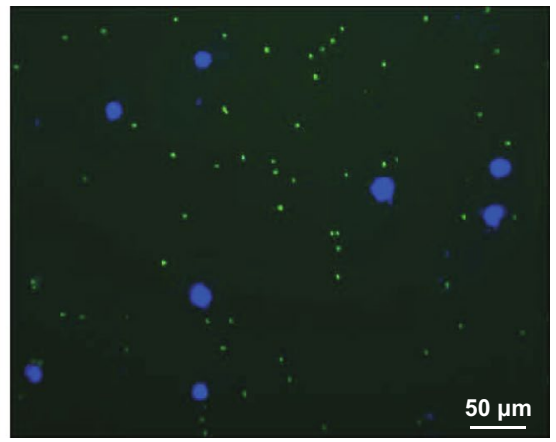

M28+U937+LDL, 30 min

d2

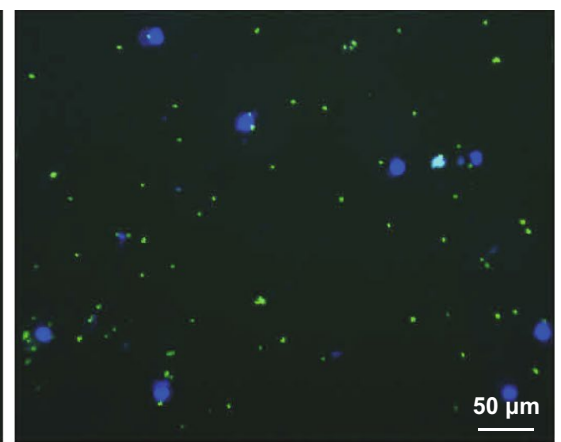

M6+U937+LDL, $30 \mathrm{~min}$ a3

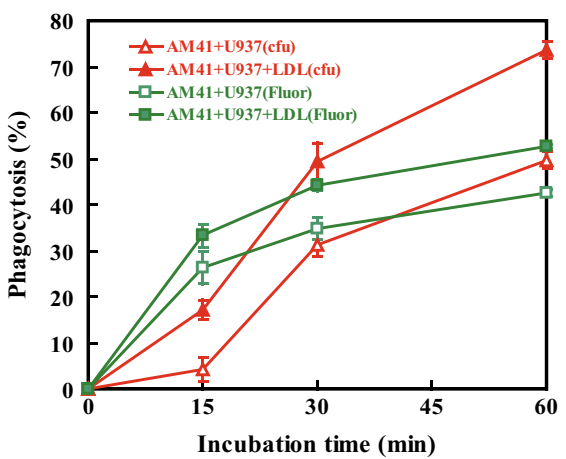

a3

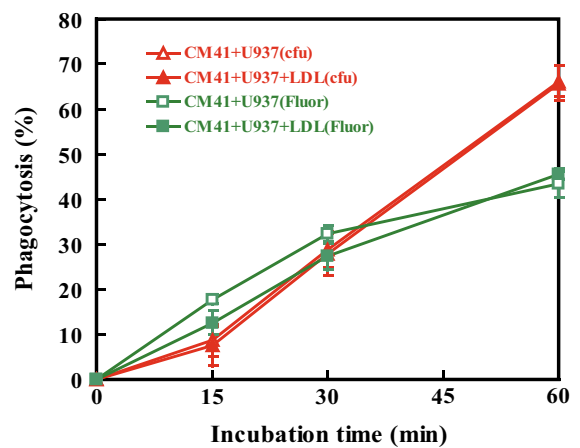

c3

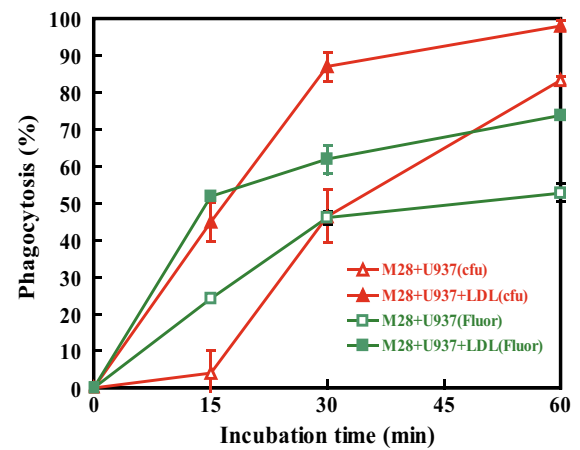

d3

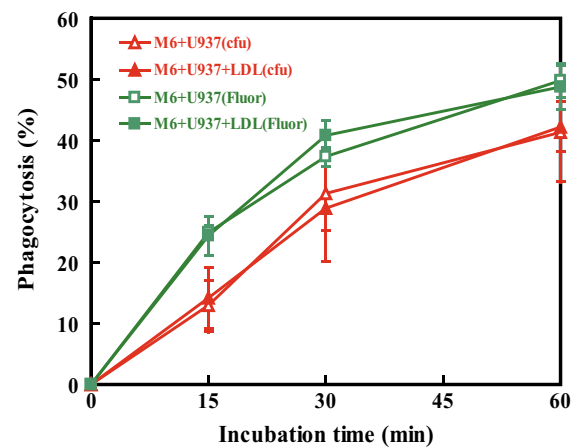


4Fig. 1 LDL-mediated phagocytosis of four GAS strains. After 30-min incubation of U937 cells with FITC-labeled GAS DAPI was added and co-cultivated for $10 \mathrm{~min} ; 5 \mathrm{ml}$ of sample was plated onto slides, and GAS (green) and DAPI-stained U937 cell nuclei (blue) were imaged, respectively, at $\times 200$ magnification on fluorescent microscope. a1, b1, c1 and d1 refer to control group, including AM41, CM41, M28 and M6-type GAS without LDL, whereas a2, b2, c2 and $\mathbf{d 2}$ stand for the treatments with LDL. The colony-forming units (cfu) of GAS in the mixture were determined by plating diluted samples onto THY agar plates after 15-, 30- and 60-min co-culture of FITC-GAS and U937 cells in the presence or absence of LDL. The phagocytosis rates were expressed as $100 \% \times$ (cfu of addition - cfu after co-culture)/cfu of addition. The red lines in $\mathbf{a 3}, \mathbf{b 3}, \mathbf{c 3}$ and $\mathbf{d} \mathbf{3}$ refer to the phagocytosis rates calculated upon cfu. Bacterial fluorescence intensity was monitored at 15, 30 and $60 \mathrm{~min}$ of incubation, and $200 \mu \mathrm{l}$ of sample was pipetted into the 96-well microplate and measured at $\lambda=495 \mathrm{~nm}$ (excitation) and $\lambda=519 \mathrm{~nm}$ (emission) using a fluorescence plate reader. The phagocytosis rates were expressed as $100 \% \times$ (initial absorbance value - absorbance value after co-culture)/initial absorbance value. The green lines in $\mathbf{a 3}, \mathbf{b 3}$, $\mathbf{c} 3$ and $\mathbf{d} \mathbf{3}$ refer to the phagocytosis rates calculated upon fluorescence (fluor)

with colony counts, because the intracellular FITC-labeled bacteria still fluoresced faintly. The opsonic phagocytosis was further demonstrated with microscopy (Fig. 1a1, LDLfree control; Fig. 1a2, LDL-positive group). In contrast to AM41-type GAS, the addition of LDL did not increase the phagocytosis rates of M41-type GAS (CMCC 32198, CM41-type GAS) by U937 cells (Fig. 1b1-b3). The results also showed that LDL significantly promoted the ingestion of M28-type GAS ( $p<0.001$, Fig. 1c1-c3) but not M6-type GAS (Fig. 1d1-d3) since the former could bind to LDL but the latter could not. In overall, our data consistently demonstrated that LDL-opsonized GAS was phagocytosed more efficiently than GAS alone. To investigate whether internalized GAS was still alive $1 \mathrm{~h}$ after phagocytosis,
U937 cells co-cultivated with GAS were lysed and plated onto THY agar plate $[9,11]$. The colony-forming unit (cfu) of the total intracellular and extracellular live bacteria was not different from that of bacteria without the lysis of U937 cells (data not shown). These results indicated that GAS was dead after ingestion by U937 cells. In addition, LDL alone promoted the growth of all tested GAS strains in the absence of U937 cells (data no shown) because LDL may act as a nutrient substance for GAS.

\section{Mechanism underlying the LDL-mediated phagocytosis of GAS}

\section{Inhibition of LDL-mediated GAS phagocytosis by recombinant $S c l 1$ ( $r S c l 1)$}

To test whether the binding of LDL to surface Scl1 of GAS was responsible for the opsonophagocytosis, rScl1 was added to the mixture of GAS, LDL and U937 cells since rScl1 could competitively inhibit the interaction of LDL with GAS (Figure S2). The results showed that rScl1 abolished the LDL-mediated opsonophagocytosis of AM41-type GAS but did not have any impact on LDLfree non-opsonic phagocytosis, which was consistent with the findings by fluorescence detection (Fig. 2a) and colony counting (Fig. 2b) after 30 min of co-cultivation of U937 cells with FITC-labeled GAS.

\section{Anti-CD36 antibody abrogated the LDL-dependent phagocytosis of GAS by $U 937$ cells}

To investigate which scavenger receptor of U937 cells was involved in LDL-mediated phagocytosis of GAS,
Fig. 2 rScl1 inhibited LDLmediated phagocytosis of GAS by U937 cells. The phagocytosis assay was carried out with similar procedure to Fig. 1 except the addition of rScl1. a The phagocytosis rates were calculated based upon cfu after 30-min incubation of U937 cells and GAS. b The phagocytosis rates were derived from fluorescence intensity (fluor) after 30-min co-culture of U937 cells and GAS
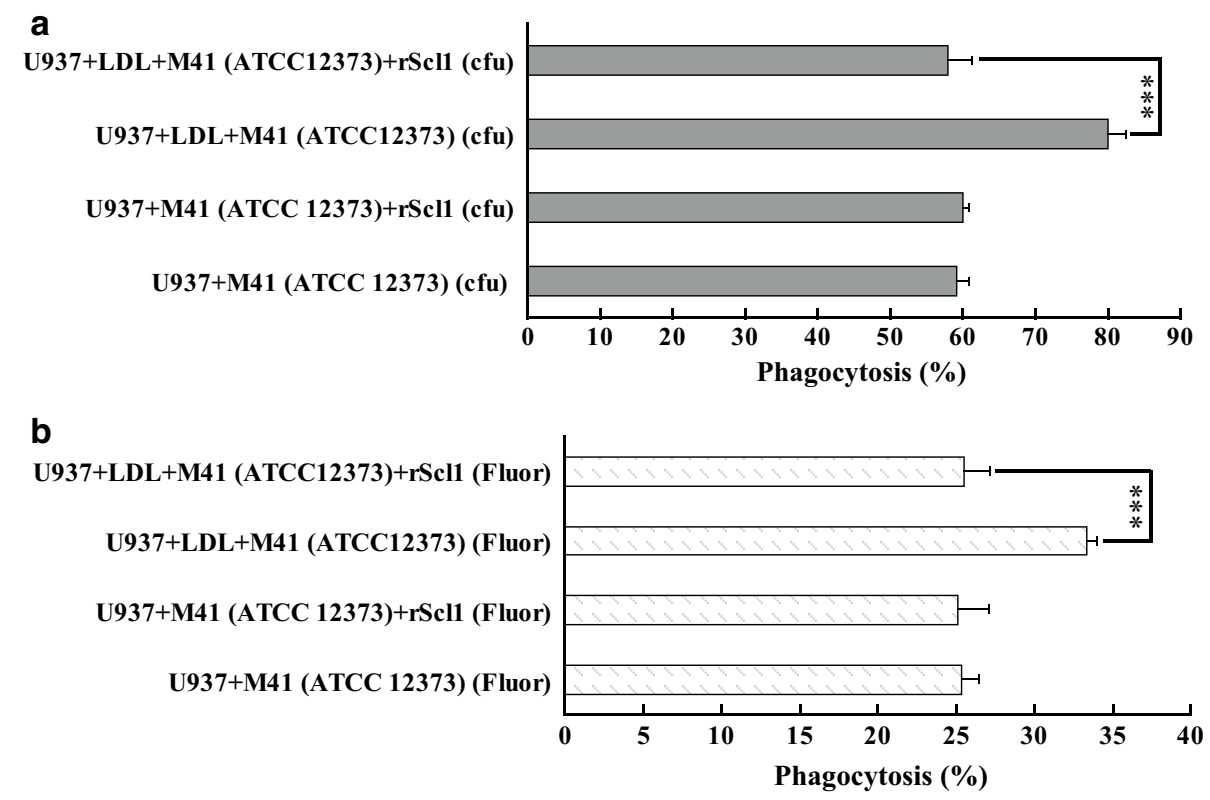
Fig. 3 Role of anti-CD36 antibody in resistance to phagocytosis of GAS by U937 cells. The phagocytosis assay was carried out with similar procedure of anti-CD36 antibody or anti-CD4 antibody. Percent of control $=($ viable GAS in other treatments/viable GAS of U937 cells and GAS group) $\times 100$ was determined after 30-min co-culture and calculated from cfu (a) and fluorescence intensity (b), respectively to Fig. 1 except the addition

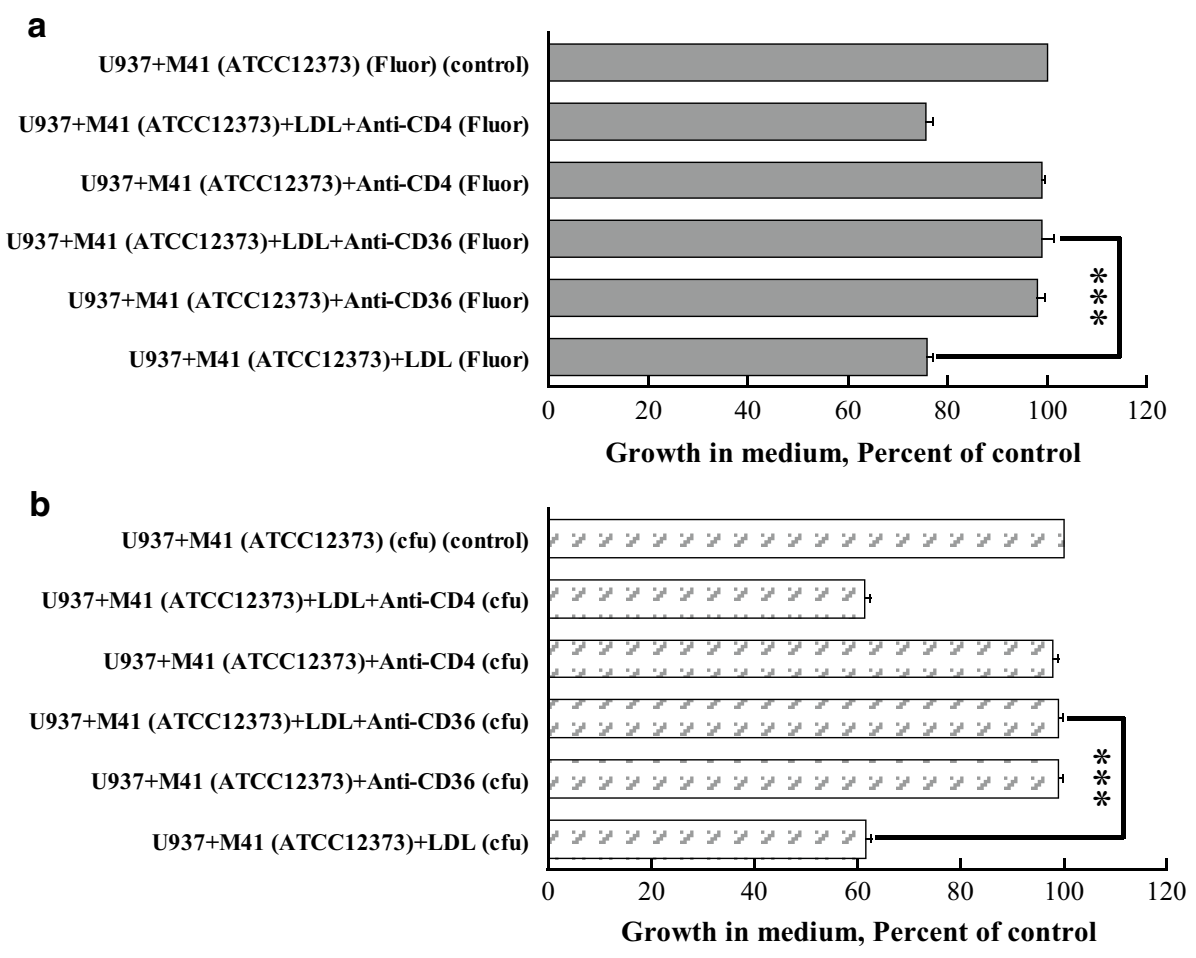

b
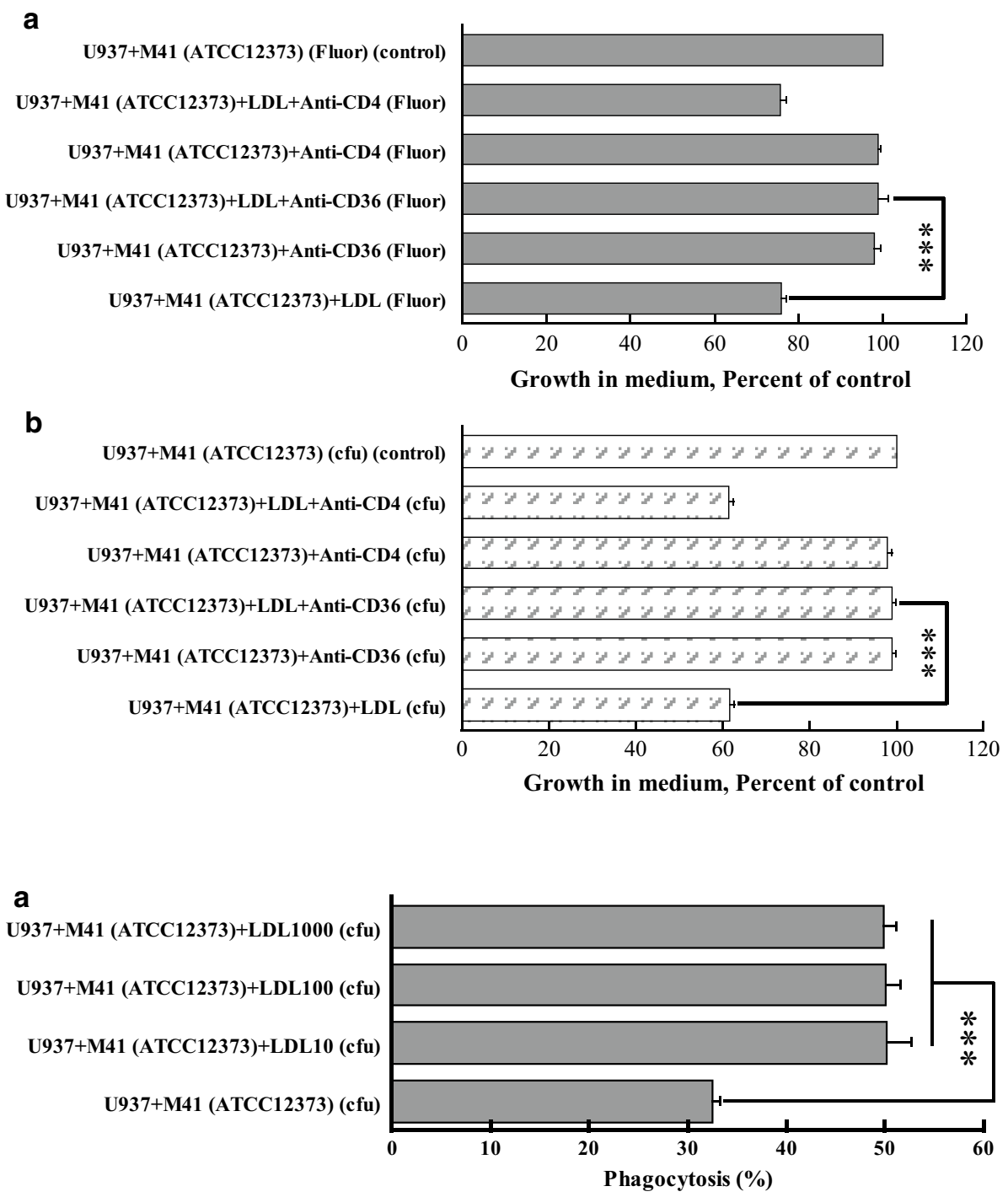

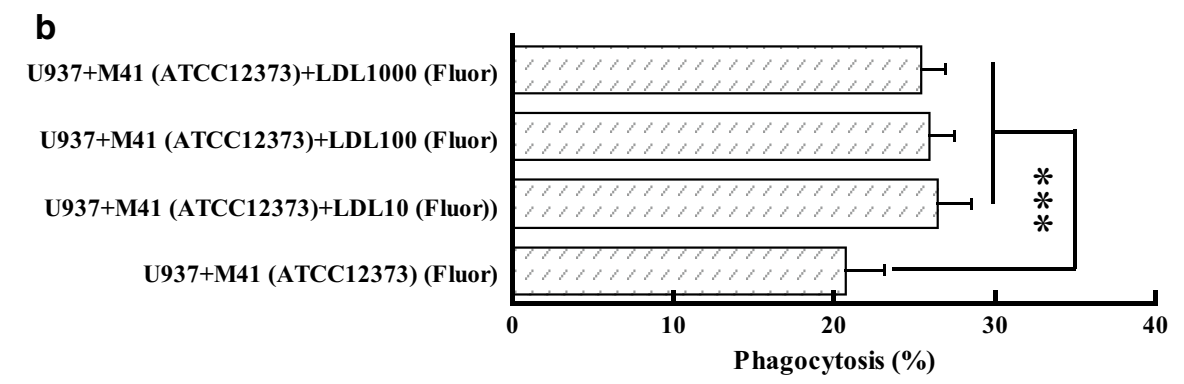

Fig. 4 Effect of LDL concentration on the opsonic phagocytosis of GAS by U937 cells. The phagocytosis assay was carried out with similar procedure to Fig. 1 except the variation of LDL concentration from 10 to $1000 \mu \mathrm{g} / \mathrm{ml}$. a The phagocytosis rates were calculated based upon cfu after 30-min incubation of U937 cells and GAS. b The phagocytosis rates were derived from fluorescence intensity (fluor) after 30-min coculture of U937 cells and GAS monoclonal anti-CD36 antibody or an irrelevant antiCD4 antibody was added into the culture. In the presence of anti-CD36 antibody, extracellular bacterial cfu and fluorescence intensity of U937 cells, LDL and GAS culture were increased to the same level as the control (Fig. 3a, b). In contrast, the addition of antiCD4 antibody did not increase the extracellular bacterial growth (Fig. 3a, b). Therefore, anti-CD36 antibody abrogated the LDL-dependent phagocytosis of AM41type GAS but did not decrease the LDL-independent phagocytosis. On the other hand, anti-CD4 antibody did not have any influence on either LDL-dependent or LDL-independent phagocytosis. These results indicated that CD36 was responsible for the recognization of LDL-GAS complex, leading to the rapid uptake of GAS by monocyte. 


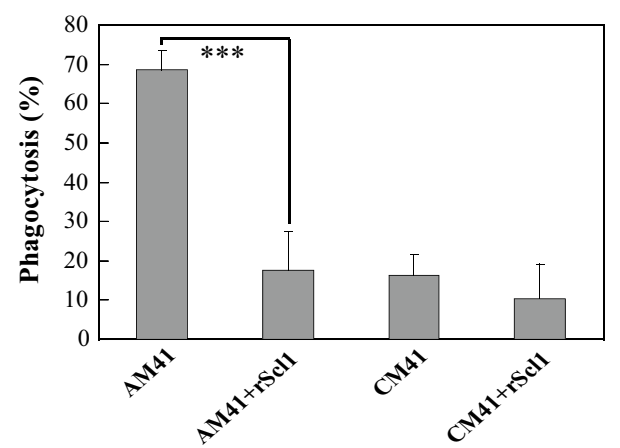

Fig. 5 Phagocytosis of GAS in human blood. The $200 \mu \mathrm{l}$ aliquot of fresh blood was pre-incubated with $50 \mu \mathrm{rScl} 1$ or PBS at $37^{\circ} \mathrm{C}$ under agitation $(170 \mathrm{rpm})$ for $1 \mathrm{~min}$. A $100 \mu \mathrm{l}$ aliquot of AM41-type or CM41-type GAS $\left(\sim 10^{3} \mathrm{cfu} / \mathrm{ml}\right)$ was added into the blood and agitated at $37{ }^{\circ} \mathrm{C}$ for $15 \mathrm{~min}$. The colony-forming unit (cfu) was determined by plating samples on THY agar plates. The phagocytosis rates were expressed as $100 \% \times(\mathrm{cfu}$ of addition $-\mathrm{cfu}$ after co-culture)/cfu of addition

\section{High concentration of $L D L$ did not inhibit the LDL-mediated phagocytosis}

CD36 is a receptor of not only LDL [8] but also oxLDL [7]. Moreover, even freshly isolated LDL from human blood still contained some oxLDL [12]. In the above experiments, $10 \mu \mathrm{g} / \mathrm{ml}$ of LDL which is less than physiological concentration (about $1000 \mu \mathrm{g} / \mathrm{ml}$ ) was used. Therefore, high concentration of LDL was tested; 100 or $1000 \mu \mathrm{g} / \mathrm{ml}$ of LDL was used to test whether LDL could competitively inhibit CD36 from recognizing LDL-GAS complex. The results showed that high concentration of LDL did not significantly decrease the phagocytosis rate even if the concentration of LDL in the culture was as high as $1000 \mu \mathrm{g} / \mathrm{ml}$ (Fig. 4a, b).

\section{LDL enhanced the phagocytosis of GAS in human blood}

To investigate whether LDL could promote the phagocytosis of GAS in human blood, we measured the viable number of AM41 and CM41-type GAS in whole blood with or without rScl1 after 15-min incubation. The phagocytic efficiency of AM41-type GAS was $69 \%$ which was significantly higher than that of CM41-type GAS (16\%) (Fig. 5). The addition of rScl1 decreased the uptake of AM41-type GAS to $19 \%$, while phagocytosis rate for CM41-type GAS was reduced to $10 \%$ (Fig. 5). Therefore, LDL also enhanced the killing of LDL-opsonized GAS in human blood.

\section{Discussion}

GAS infection is an increasing problem, with $>500,000$ deaths per year [13]. Scl1-LDL interaction was found, but the significance of the interaction especially in the host immunity has not been uncovered. To the best of our knowledge, LDL was demonstrated for the first time to be an opsonin to enhance CD36-dependent opsonic phagocytosis of GAS by monocyte and whole human blood in this study.

In fact, LDL presents anti-Klebsiella pneumoniae, $K$. pneumonia, Salmonella typhimurium, Listeria monocytogenes, Mycobacterium tuberculosis and Vibrio vulnificus capability of preventing these bacterium infections [6]. LDL but not HDL binds to prions from the brains of patients with sporadic Creutzfeldt-Jakob disease to prevent the prion further invasion [14]; Pseudomonas aeruginosa was demonstrated to bind to oxLDL but not to LDL through protein PA0122 in a previous study [15] where the significance of the oxLDL-PA0122 interaction was not addressed. Our findings in this study demonstrated that the interaction of Sc11 and LDL played an important anti-infectious role. In fact, LDL may be an opsonin to enhance the phagocytosis of Yersinia pestis by monocyte due to the binding of LDL to $Y$. pestis [16]. However, pure pH6-Ag (similar to rScl1) can also competitively inhibit the binding of LDL to $Y$. pestis to abrogate LDL-mediated opsonophagocytosis of $Y$. pestis.

Oxidation of LDL may be a more important response of the host against infections; therefore, whether there exists an interaction of OxLDL with Sc11 and the implication of this interaction in the host innate immunity is worth pursuing.

We found for the first time that CD36 could be a much higher affinity receptor of the LDL opsonin receptor, because the results demonstrated that once LDL binds to GAS, LDL-GAS complex was quickly recognized by CD36 and phagocytosed by monocyte. The findings are not in line with the previous findings in other studies where scavenger receptors, including CD36, were previously thought to only mediate the non-opsonic phagocytosis of bacteria by monocyte/macrophage $[17,18]$.

Based upon our findings, monocyte or macrophage might ingest bacterium-LDL complex to protect against infections, which might result in the formation of foam cell, cholesterol-laden macrophage since LDL cholesterol is considered as a major factor in atherosclerosis development [19]. So far, the validity of the hypothesis that infection contributes to atherosclerosis has not been definitively established although the evidence is becoming compelling [20-22]. Our findings might shed a new light on the correlation between infections and atherosclerosis due to the double-edged sword property of LDL.

Severe invasive streptococcal infections are often associated with systemic dissemination, which reflects the diverse abilities of GAS to avoid eradication by phagocytic defenses. Therefore, GAS can be isolated from the 
bloodstream in most patients with invasive infection [23]. Polymorphonuclear neutrophils are the most important phagocytes in the blood; GAS, however, can avoid the killing by PMNs and survive within these cells [24]. Thus, LDL-mediated opsonophagocytosis of GAS by monocyte found in this study is particularly important for the rapid clearance of GAS by host since GAS inside the monocyte was killed within $1 \mathrm{~h}$.

Our experiments were performed only in in vitro and ex vivo systems since GAS is a strictly human pathogen [25] and only a very few streptococcal strains can establish infection in mice. On the other hand, knockout of ApoB100 is lethal to animal. Therefore, a careful and profound strategy to further confirm the findings in this study in an in vivo system should be endeavored. Nevertheless, our findings that LDL was identified to be an opsonin to enhance CD36 mediated phagocytosis of GAS by monocyte and whole human blood may provide clues for anti-GAS infection and atherosclerosis prevention strategies.

Acknowledgments We thank En Liu and Yang Wang (Research Center of Plasma Lipoprotein Immunology) for technical assistance.

\section{Compliance with ethical standards}

Conflict of interest The authors declare no competing financial interests.

Open Access This article is distributed under the terms of the Creative Commons Attribution 4.0 International License (http://creativecommons.org/licenses/by/4.0/), which permits unrestricted use, distribution, and reproduction in any medium, provided you give appropriate credit to the original author(s) and the source, provide a link to the Creative Commons license, and indicate if changes were made.

\section{References}

1. Cunningham MW (2000) Pathogenesis of group A streptococcal infections. Clin Microbiol Rev 13(3):470-511

2. McMillan DJ, Dreze PA, Vu T, Bessen DE, Guglielmini J, Steer AC, Carapetis JR, Van Melderen L, Sriprakash KS, Smeesters PR, Grp MPS (2013) Updated model of group A Streptococcus $\mathrm{M}$ proteins based on a comprehensive worldwide study. Clin Microbiol Infect 19(5):E222-E229

3. Han R, Zwiefka A, Caswell CC, Xu Y, Keene DR, Lukomska E, Zhao Z, Hook M, Lukomski S (2006) Assessment of prokaryotic collagen-like sequences derived from streptococcal Scl1 and Scl2 proteins as a source of recombinant GXY polymers. Appl Microbiol Biotechnol 72(1):109-115

4. Caswell CC, Han R, Hovis KM, Ciborowski P, Keene DR, Marconi RT, Lukomski S (2008) The Scl1 protein of M6-type group A Streptococcus binds the human complement regulatory protein, factor $\mathrm{H}$, and inhibits the alternative pathway of complement. Mol Microbiol 67(3):584-596

5. Han R, Caswell CC, Lukomska E, Keene DR, Pawlowski M, Bujnicki JM, Kim JK, Lukomski S (2006) Binding of the low-density lipoprotein by streptococcal collagen-like protein Scl1 of Streptococcus pyogenes. Mol Microbiol 61(2):351-367

6. Han R (2010) Plasma lipoproteins are important components of the immune system. Microbiol Immunol 54(4):246-253

7. Murphy JE, Tedbury PR, Homer-Vanniasinkam S, Walker JH, Ponnambalam S (2005) Biochemistry and cell biology of mammalian scavenger receptors. Atherosclerosis 182(1):1-15

8. Calvo D, Gómez-Coronado D, Suárez Y, Lasunción MA, Vega MA (1998) Human CD36 is a high affinity receptor for the native lipoproteins HDL, LDL, and VLDL. J Lipid Res 39(4):777-788

9. Adolph S, Fuhrmann H, Schumann J (2012) Unsaturated fatty acids promote the phagocytosis of $P$. aeruginosa and $R$. equi by RAW264.7 macrophages. Curr Microbiol 65(6):649-655

10. Gao Y, Liang C, Zhao R, Lukomski S, Han R (2010) The Scl1 of M41-type group A Streptococcus binds the high-density lipoprotein. FEMS Microbiol Lett 309(1):55-61

11. Medina E, Goldmann O (2011) In vivo and ex vivo protocols for measuring the killing of extracellular pathogens by macrophages. Curr Protoc Immunol. Chapter 14: Unit 14. 19. 1-17

12. Frostegård J, Nilsson J, Haegerstrand A, Hamsten A, Wigzell H, Gidlund M (1990) Oxidized low density lipoprotein induces differentiation and adhesion of human monocytes and the monocytic cell line U937. Proc Natl Acad Sci USA 87(3):904-908

13. Cohen-Poradosu R, Kasper DL (2007) Group A Streptococcus epidemiology and vaccine implications. Clin Infect Dis 45(7):863-865

14. Safar JG, Wille H, Geschwind MD, Deering C, Latawiec D, Serban A, King DJ, Legname G, Weisgraber KH, Mahley RW, Miller BL, Dearmond SJ, Prusiner SB (2006) Human prions and plasma lipoproteins. Proc Natl Acad Sci USA 103(30):11312-11317

15. Rao J, DiGiandomenico A, Unger J, Bao Y, PolanowskaGrabowska RK, Goldberg JB (2008) A novel oxidized low-density lipoprotein-binding protein from Pseudomonas aeruginosa. Microbiology 154(2):654-665

16. Makoveichuk E, Cherepanov P, Lundberg S, Forsberg A, Olivecrona G (2003) pH6 antigen of Yersinia pestis interacts with plasma lipoproteins and cell membranes. J Lipid Res 44(2):320-330

17. Stuart LM, Deng J, Silver JM, Takahashi K, Tseng AA, Hennessy EJ, Ezekowitz RA, Moore KJ (2005) Response to Staphylococcus aureus requires CD36-mediated phagocytosis triggered by the $\mathrm{COOH}$-terminal cytoplasmic domain. J Cell Biol 170(3):477-485

18. Ofek I, Goldhar J, Keisari Y, Sharon N (1995) Nonopsonic phagocytosis of microorganisms. Annu Rev Microbiol 49(1):239-276

19. Tomkin GH, Owens D (2012) LDL as a cause of atherosclerosis. Open Atheroscler Thromb J 5:13-21

20. Levi M, van der Poll T, Schultz M (2012) Infection and inflammation as risk factors for thrombosis and atherosclerosis. Semin Thromb Hemost 38(5):506-514

21. Chatzidimitriou D, Kirmizis D, Gavriilaki E, Chatzidimitriou M, Malisiovas N (2012) Atherosclerosis and infection: is the jury still not in? Future Microbiol 7(10):1217-1230

22. Ravnskov U, McCully KS (2012) Infections may be causal in the pathogenesis of atherosclerosis. Am J Med Sci 344(5):391-394

23. Kwinn LA, Nizet V (2007) How group A Streptococcus circumvents host phagocyte defenses. Future Microbiol 2(1):75-84

24. Medina E, Goldmann O, Toppel AW, Chhatwal GS (2003) Survival of Streptococcus pyogenes within host phagocytic cells: a pathogenic mechanism for persistence and systemic invasion. $\mathbf{J}$ Infect Dis 187(4):597-603

25. Bessen DE (2009) Population biology of the human restricted pathogen, Streptococcus pyogenes. Infect Genet Evol 9(4):581-593 